\title{
Multiple Brain Abscesses of Gynecologic Origin Caused by Fusobacterium nucleatum
}

\section{Abcès multiples d'origine gynécologique dus à Fusobacterium nucleatum}

\author{
K. Rachdi $\cdot$ V. Legros $\cdot$ S. Mestrallet $\cdot$ B. Just $\cdot$ P. Mateu
}

Received: 10 December 2013; Accepted: 22 February 2014

(C) SRLF et Springer-Verlag France 2014

To the Editor,

On December 04, 2012, a 47-year-old immunocompetent woman, with a past history of obesity, chronic alcoholism, diverticulitis, and intrauterine device (IUD) in place for at least fifteen years, was admitted to the emergency department because of confusion, agitation, and vomiting lasting for several hours. In June 2012, an ovarian mass of possible functional origin was diagnosed using ultrasounds at her usual gynecologic examination. In November 2012, due to the sudden onset of headaches and tremor, she was referred to the gynecologic emergency ward. Gynecologic examination was normal. Her complaints were considered as anxiety disorders. IUD withdrawal was planned for her next medical visit. In the following days, she continuously suffered from headaches and used non-steroidal anti-inflammatory drugs (NSAID) along with paracetamol as self-medication. On admission in the emergency department, severe intracranial hypertension was immediately suspected while physical examination ruled out meningeal irritation. The patient was intubated, mechanically ventilated, sedated, and transferred to the intensive care unit (ICU). The IUD was removed and cultured. Computed tomography (CT) scan showed several intracerebral hypodense lesions with loss of cortical sulci of the convexity and cerebral herniation. After the injection of contrast material, four round lesions with contrast enhancement and important peri-lesional edema were evidenced (Fig. 1). No sign of cerebral venous thrombosis was found.

All the clinical, biological, and radiological investigations searching for a primary cancer were negative. Pelvic CT scan revealed the presence of salpingitis and ovarian cysts of

\footnotetext{
K. Rachdi · V. Legros · B. Just · P. Mateu $(\bowtie)$

Intensive Care Unit, Manchester hospital, 45, avenue de

Manchester, F-08000 Charleville-Mézières, France

e-mail : p.mateu@orange.fr

S. Mestrallet

Infectiology Unit, Manchester hospital, 45, avenue de

Manchester, F-08000 Charleville-Mézières, France
}

functional origin as evidenced by the histological examination of a biopsy sample. The anaerobic blood cultures obtained before the administration of antibiotics were positive for Fusobacterium nucleatum, sensitive to all major antibiotics. Lumbar puncture was not performed due to the presence of cerebral herniation. Neurosurgical drainage was not indicated. Bacteriological culture suggested IUD colonization by $F$. nucleatum. No other origin for the infection was found. All other investigations were negative, including ear, nose, throat (ENT) examination and dental imaging. Probabilistic treatment combining $4 \mathrm{~g} \mathrm{x} 4 / \mathrm{d}$ cefotaxime, $16 \mathrm{~g} / \mathrm{d}$ fos-

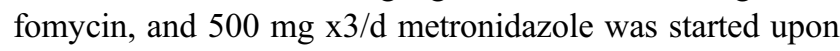
admission, in association with $120 \mathrm{mg} / \mathrm{d}$ methylprednisolone. Later, fosfomycin was stopped and cefotaxime continued to treat simultaneous hospital-acquired Hafnia alvei pneumonia. Initial seizures were treated with levetiracetam and phenobarbital. Intracranial hypertension progressively decreased without persistent neurological deficit. Significant clinical improvement was observed after eight days, allowing weaning from mechanical ventilation and extubation. Only fatigue and mild regressive frontal behavior disorders were present. Brain CT scan performed on day 13 and day 45 confirmed the regression of all the nodular lesions and the decrease of the peri-lesional edema. After one month, the patient was discharged from hospital with normal cognitive functions and autonomy.

\section{Discussion}

The infectious origin of our patient's brain abscesses was supported by her recovery with only antibiotics. Initially, due to the presence of an ovarian mass, a metastatic tumor was hypothesized. However, all the investigations searching for a primary cancer were negative. Thus, F. nucleatum, found in blood cultures, was considered as the possible cause of brain abscesses, although no direct evidence was 


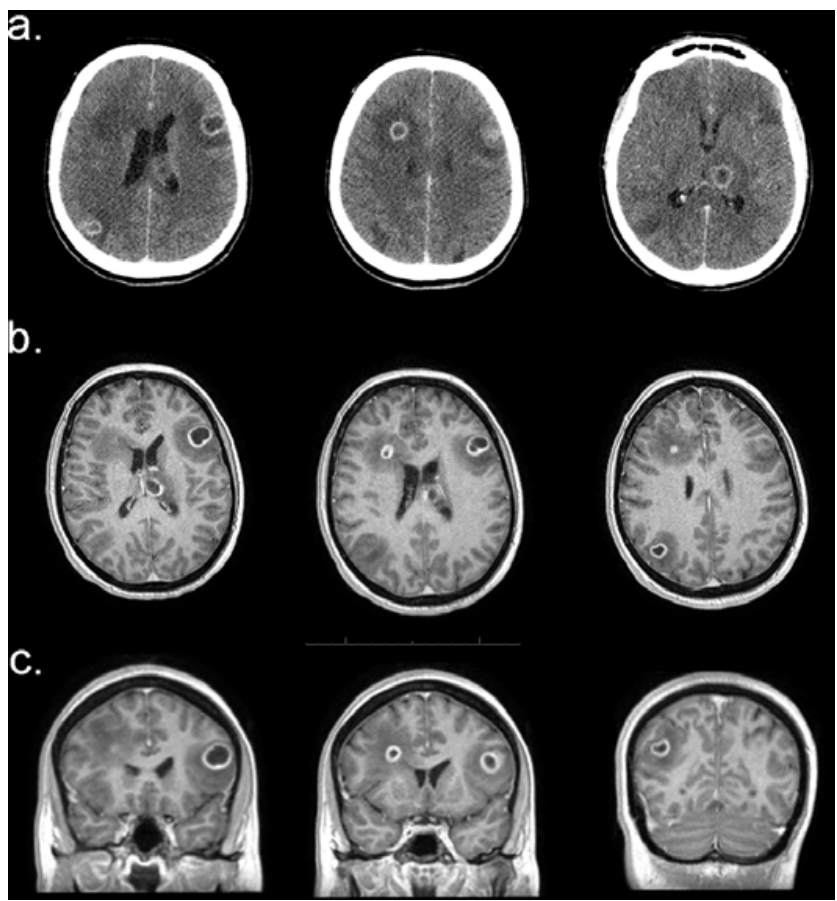

Fig. 1 Multiple cortical and subcortical ring-enhancing lesions, with peri-lesional edema. a. Initial cerebral injected CT scan; b. T1 gadolinium injected MRI (axial); c. T1 gadolinium injected MRI (coronal)

obtained and a polybacterial infection could not be definitively ruled out.

Among Fusobacterium spp., F. nucleatum is the most frequently isolated species in humans [1]. This Gram-negative anaerobic bacillus is a rarely pathogenic ENT-commensal bacterium. Most of $F$. nucleatum-attributed infections are localized in the ENT, including gingival and periodontal infections. Here, the infection seemed to have a gynecologic origin, caused by the IUD in place for over fifteen years based on the identification of $F$. nucleatum on the IUD despite the difficulties to obtain additional subcultures. Moreover, the initial CT scan showed infection of the Fallopian tubes that could be considered as the initial Fusobacterium infection leading to bacteremia. Several cases of gynecologic F. nucleatum infections have been described [2]. Most of them are intra-amniotic infections associated with preterm labor [3]. An alternative hypothesis for the initial infection could be a sigmoiditis, as diagnosed one year earlier by her general physician and treated with antibiotics. The literature reports several cases of $F$. nucleatum-related digestive infections [2]. However, brain abscesses remain exceptional [4-7]. In the absence of immunodeficiency, onset of such multiple brain abscesses could not be easily explained, unless considering the use of NSAID by the patient as a possible risk factor.

Given the number of abscesses, their location, the good response to treatment, and the regression of all neurological features, no neurosurgical puncture was performed in our patient. Cefotaxime and metronidazole combination in addition to neurosurgery was reported to be effective to treat cerebral abscesses, due to their excellent in situ distribution [7]. Interestingly, several other cases of multiple F. nucleatum-related brain abscesses were successfully treated with antibiotics alone $[4,8]$. Like in our case, antibiotics without systematic neurosurgical drainage appear to be a safe strategy to obtain a final favorable outcome.

Acknowledgments We thank Ms. Martine-Cécile MeunierJust for her assistance in translating the manuscript.

Conflict of interest: K. Rachdi, V. Legros, S. Mestrallet, B. Just et P. Mateu have no conflict of interest to declare.

\section{References}

1. Citron DM (2002) Update on the taxonomy and clinical aspects of the genus Fusobacterium. Clin Infect Dis 35:S22-S7

2. Huggan PJ, Murdoch DR (2008) Fusobacterial infections: clinical spectrum and incidence of invasive disease. J Infect 57:283-9

3. Mikamo H, Kawazoe K, Sato Y, Tamaya T (1998) Preterm labor and bacterial intra-amiotic infection: arachidonic acid liberation by phospholipase A2 of Fusobacterium nucleatum. Am J Obstet Gynecol 179:1579-82

4. Heckmann JG, Lang CJG, Hartl H, Tomandl B (2003) Multiple brain abscesses caused by Fusobacterium nucleatum treated conservatively. Can J Neurol Sci 30:266-8

5. Hsieh MJ, Chang WN, Lui CC, et al (2007) Clinical characteristics of fusobacterial brain abscess. Jpn J Infect Dis 60:40-4

6. Han XY, Weinberg JS, Prabhu SS, et al (2003) Fusobacterial brain abscess: a review of five cases and an analysis of possible pathogenesis. J Neurosurg 99:693-700

7. Sjölin J, Lilja A, Eriksson N, et al (1993) Treatment of brain abscess with cefotaxime and metronidazole: prospective study on 15 consecutive patients. Clin Infect Dis 17:857-63

8. Boom WH, Tuazon CU (1985) Successful treatment of multiple brain abscesses with antibiotics alone. Rev Infect Dis 7:189-99 